\title{
PYMEs, competitividad y el CAFTA
}

Rick van der Kamp ${ }^{1}$

1. asesor de estrategia Nitlapán - UCA, programa Fomento a la Pequeña Empresa

e-mail: rickvanderkamp@gmail.com

\section{Recibido: mayo 2006 / Aceptado: junio 2006}

ESTE ARTÍCULO INVESTIGA EL TEMA DE LA COMPETITIVIDAD del sector PYME (pequeñas y medianas empresas) en Nicaragua, y lo vincula con el entorno de libre comercio acentuado por la entrada en vigencia del CAFTA. Se define el concepto de competitividad y sus componentes, y presenta información sobre la competitividad de Nicaragua comparada con otros países. Explorando los distintos componentes de la competitividad, también presenta estadísticas sobre la productividad laboral y el clima de negocios para llegar a un posicionamiento del país en el entorno mundial. El artículo explora el contenido del tratado de libre comercio CAFTA, enfocándose en los cambios que genera para el comercio internacional de Nicaragua. Además, usando información sobre importaciones y exportaciones, se tratará de estimar el impacto que tendrá el tratado en el sector PYME, las empresas grandes, el gobierno, y la población de Nicaragua. Finalmente, se discute el desafío de la globalización, y las estrategias más efectivas que las PYMEs aprovechen nuevas oportunidades generadas.

Palabras clave: PYMEs, pequeña empresa - Nicaragua, competitividad, productividad, clima de negocios, desarrollo económico, comercio internacional, libre comercio, cadenas productivas.

\section{Introducción}

Este artículo presenta los resultados y conclusiones más importantes de un estudio más amplio sobre el sector PYME en Nicaragua, ejecutado dentro del programa Fomento a la Pequeña Empresa (FPE) de Nitlapán - UCA. El propósito del estudio era generar una visión completa del estado actual del sector, y de los mayores problemas y oportunidades que enfrenta el sector hoy en día. Esta visión, en una segunda etapa, ha servido para determinar la estrategia más adecuada para el programa FPE en brindar servicios y productos de valor agregado para el sector PYME. Paralelamente, se ha continuado con la actualización y agregación de información al estudio, para que en si mismo podrá servir como documento de referencia para cualquier persona interesada en las pequeñas empresas de Nicaragua.

El estudio tiene un enfoque cuantitativo, dado la gran falta de este tipo de información sobre el sector PYME. Además de usar las estadísticas disponibles (en mayor parte del Banco Central de Nicaragua y de organizaciones multilaterales), el estudio también ha utilizado 
fuentes secundarias en la forma de las varias publicaciones y analices ya disponibles sobre el tema. Por fin, el estudio ha beneficiado importantemente de la experiencia y información disponible dentro del programa FPE, donde la interacción directa con la población meta (pequeñas empresas) genera información cualitativa y cuantitativa no disponible en fuentes académicas.

\section{PYMEs y competitividad}

Tal vez el tema más aplicado para países en desarrollo ha sido el concepto de competitividad. Desde la definición de Porter - la competitividad es definida por la productividad en que un país utiliza sus recursos humanos, financieros y naturales - se desarrolló un marco teórico más detallado, que presentaba el concepto de "competitividad sistémica". Este concepto reconoce factores que influyen a la competitividad en cuatro niveles: meta, macro, meso y micro.

El diagrama, abajo, presenta una estilización de los factores de la competitividad sistémica. Para la situación del sector PYME en Nicaragua, es importante notar que la competitividad no es meramente determinada por el sistema productivo de una empresa, sino impactada por factores culturales, macro-económicos, educacionales e infraestructurales, entre otros. Esto significa que en una consideración de la competitividad del sector PYME nacional, se debería tomar en cuenta estos factores, tal como el desempeño productivo de las empresas mismas.

\section{Factores determinantes de la competitividad sistémica}

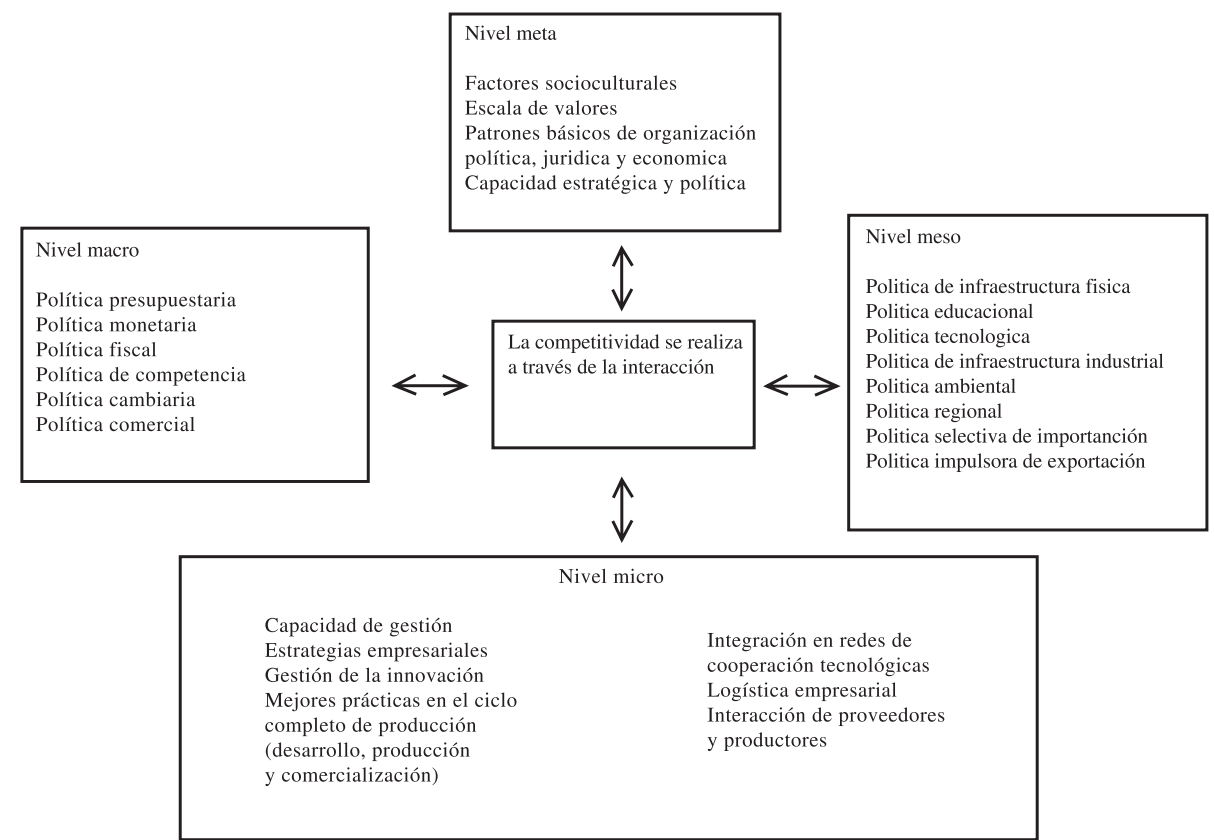

Fuente: CEPAL (2001) 


\section{Nicaragua y la competitividad internacional}

El tema de la competitividad es tal vez el más mencionado en las discusiones y programas de apoyo para el sector PYME en Nicaragua. Sin embargo, no siempre se explicita los detalles del concepto de competitividad, lo cual hace más difícil una evaluación factual del concepto. El Foro Económico Mundial (FEM, World Economic Forum), líder en las discusiones sobre el tema de la competitividad entre países en los últimos años, define competitividad como "la colección de factores, políticas y instituciones que determinan el nivel de productividad de un país y, por ende, determinan el nivel de prosperidad que puede alcanzar una economía”.

La misma organización también provee datos y clasificaciones que indican la posición de los países investigados en términos de su competitividad. Entre ellos se distingue dos rankings: el de competitividad de crecimiento (económico) y el de competitividad de negocios. El primero se define por la calidad de la innovación y adaptación tecnológica, las instituciones públicas y el ámbito macroeconómico (lo cual incluye el uso de finanzas públicas, política de tasa de cambio). La competitividad empresarial, según el FEM, es determinada tanto por la calidad de operaciones y estrategia de las empresas, como por la calidad del entorno de negocios de un país. La tabla de abajo muestra esos rankings y presenta la posición de Nicaragua en ellos.

La tabla demuestra la posición baja del país tal como sus negocios en el entorno internacional. Los indicadores implican que el país es específicamente débil en su entorno macroeconómico y en las operaciones y la estrategia de sus empresas. De los países de Latinoamérica, se nota la posición elevada de Chile y El Salvador (relativo a su PIB per capita), y en Asia se muestra el gran potencial de los dos gigantes: China e India, ambos en posiciones relativamente altas en la tabla.

Cuadro 1. Índice de Competitividad de Crecimiento y de Negocios, 2005.

\begin{tabular}{|c|c|c|c|c|c|c|}
\hline País & GCI 2005 & Score & GCI 2004 & $\begin{array}{c}\text { Tecno- } \\
\text { logía }\end{array}$ & $\begin{array}{l}\text { Institu- } \\
\text { ciones }\end{array}$ & $\begin{array}{c}\text { Macro- } \\
\text { econ. }\end{array}$ \\
\hline Finlandia & 1 & 5.94 & 1 & 2 & 5 & 4 \\
\hline Estados Unidos & 2 & 5.81 & 2 & 1 & 18 & 23 \\
\hline Singapore & 6 & 5.48 & 7 & 10 & 4 & 1 \\
\hline Icelandia & 7 & 5.48 & 10 & 9 & 3 & 11 \\
\hline Chile & 23 & 4.91 & 22 & 35 & 22 & 15 \\
\hline China & 49 & 4.07 & 46 & 64 & 56 & 33 \\
\hline India & 50 & 4.04 & 55 & 55 & 52 & 50 \\
\hline Mauritius & 52 & 4.00 & 49 & 47 & 55 & 59 \\
\hline México & 55 & 3.92 & 48 & 57 & 71 & 43 \\
\hline El Salvador & 56 & 3.86 & 53 & 70 & 54 & 57 \\
\hline Costa Rica & 64 & 3.72 & 50 & 56 & 58 & 82 \\
\hline Brasil & 65 & 3.69 & 57 & 50 & 70 & 79 \\
\hline Indonesia & 74 & 3.53 & 69 & 66 & 89 & 64 \\
\hline Honduras & 93 & 3.18 & 97 & 95 & 88 & 89 \\
\hline Guatemala & 97 & 3.12 & 80 & 96 & 107 & 81 \\
\hline Nicaragua & 99 & 3.08 & 95 & 102 & 82 & 110 \\
\hline Ecuador & 103 & 3.01 & 90 & 100 & 113 & 80 \\
\hline Zimbabwe & 109 & 2.89 & 99 & 98 & 80 & 117 \\
\hline Bangladesh & 110 & 2.86 & 102 & 101 & 117 & 83 \\
\hline Chad & 117 & 2.37 & 104 & 117 & 116 & 114 \\
\hline
\end{tabular}

\begin{tabular}{|c|cc|}
\hline BCI 2005 & $\begin{array}{c}\text { Ops \& } \\
\text { Strat }\end{array}$ & $\begin{array}{c}\text { Entorno } \\
\text { negocios }\end{array}$ \\
\hline $\mathbf{2}$ & 9 & 1 \\
\hline $\mathbf{1}$ & 1 & 2 \\
\hline $\mathbf{5}$ & 14 & 5 \\
\hline $\mathbf{1 7}$ & 15 & 18 \\
\hline $\mathbf{2 9}$ & 31 & 29 \\
\hline $\mathbf{5 7}$ & 53 & 58 \\
\hline $\mathbf{3 1}$ & 30 & 31 \\
\hline $\mathbf{5 2}$ & 45 & 49 \\
\hline $\mathbf{6 0}$ & 55 & 62 \\
\hline $\mathbf{5 8}$ & 57 & 56 \\
\hline $\mathbf{5 0}$ & 34 & 53 \\
\hline $\mathbf{4 9}$ & 32 & 52 \\
\hline $\mathbf{5 9}$ & 50 & 59 \\
\hline $\mathbf{1 0 5}$ & 95 & 105 \\
\hline $\mathbf{1 0 3}$ & 83 & 104 \\
\hline $\mathbf{1 0 6}$ & 110 & 106 \\
\hline $\mathbf{1 0 7}$ & 96 & 108 \\
\hline $\mathbf{8 4}$ & 78 & 84 \\
\hline $\mathbf{1 0 0}$ & 99 & 101 \\
\hline $\mathbf{1 1 6}$ & 116 & 116 \\
\hline & & \\
\hline & & \\
\hline
\end{tabular}

Fuente: World Economic Forum, 2005 


\section{Productividad}

Dada la importancia del concepto de la productividad, será interesante comparar las estadísticas de la productividad empresarial entre varios países. La tabla de abajo presenta datos sobre producto interno, valor agregado en manufactura y costos laborales para países en varias partes del mundo. El Valor Agregado Manufacturera (VAM) por trabajador indica el valor agregado que produce cada empleado dentro del sector de manufactura (calculado como VAM total por empleo en el sector). Generalmente, el VAM por trabajador se relaciona con el PIB per cápita, con los países más ricos, produciendo más valor agregado por empleado que países más pobres. En términos relativos, lo más notable en esta columna es el valor bajo de Mauritius.

La siguiente columna presenta los costos anuales del trabajo manufacturero. Expresado como factor del PIB, otra vez se destaca Mauritius (esta vez en sentido positivo), y se nota que los países más "caros" son Zimbabwe, Nicaragua y Ecuador. Lógicamente, los países donde los empleados generan menos valor agregado deberían tener un costo más bajo del trabajo. La última columna calcula el valor agregado producido con US\$1 en costos laborales. Aquí se prueba que los países más productivos son India (US\$4.01), México (US\$3.76), Brasil (US\$3.45) y China (US\$3.31), mientras el país menos productivo es Nicaragua (US\$1.6).

La comparación con Indonesia, un país asiático con más o menos el mismo nivel de producción interna, resulta muy favorable para el archipiélago. Mientras la productividad (VAM por trabajador) era casi 1.7 veces más alta (datos de Indonesia son de 2002), los costos laborales son menos de la mitad. Esos tipos de argumentos son importantes para perfilarse como país que atrae la inversión extranjera, un perfil que según los datos de la tabla no resulta totalmente favorable para Nicaragua.

Cuadro 2. Productividad y costos de labor, varios países, desde 2000*

\begin{tabular}{|c|c|c|c|c|c|}
\hline (2000-03*, en US\$) & PIB p.c. & $\begin{array}{c}\text { Valor Agregado } \\
\text { Manufacturera } \\
\text { (por trab.) }\end{array}$ & $\begin{array}{l}\text { Costo Labor } \\
\text { Manufacturera }\end{array}$ & $\begin{array}{c}C L, \% \text { de } \\
\text { PBI }\end{array}$ & $V A M$, con $\$ 1$ \\
\hline Estados Unidos & 37,425 & 116,750 & 45,540 & 1.22 & $\$ 2.56$ \\
\hline México & 6,122 & 41,156 & 10,944 & 1.79 & $\$ 3.76$ \\
\hline Mauritius & 4,613 & 7,297 & 3,612 & 0.78 & $\$ 2.02$ \\
\hline Panamá & 4,124 & 21,495 & 9,876 & 2.39 & $\$ 2.18$ \\
\hline Brazil & 2,788 & 19,559 & 5,676 & 2.04 & $\$ 3.45$ \\
\hline El Salvador & 2,249 & 11,587 & 3,596 & 1.60 & $\$ 3.22$ \\
\hline Ecuador & 2,116 & 19,570 & 6,636 & 3.14 & $\$ 2.95$ \\
\hline Guatemala & 2,020 & n.a. & 3,480 & 1.72 & - \\
\hline Jordan & 1,839 & 13,589 & 4,356 & 2.37 & $\$ 3.12$ \\
\hline China & 1,109 & 6,985 & 2,112 & 1.90 & $\$ 3.31$ \\
\hline Indonesia & 806 & 7,056 & 1,142 & 1.42 & $\$ 6.18$ \\
\hline Nicaragua & 785 & 4,197 & 2,664 & 3.39 & $\$ 1.58$ \\
\hline India & 555 & 5,053 & 1,260 & 2.27 & $\$ 4.01$ \\
\hline Bangladesh & 419 & 2,066 & 852 & 2.03 & $\$ 2.42$ \\
\hline Zimbabwe & 388 & n.a. & 1,572 & 4.05 & - \\
\hline
\end{tabular}

* Información más recién disponible (2000 a 2003); Bangladesh 1996.

Fuente: Estadísticas on-line, UNIDO y ILO 
Es importante tomar en cuenta que estas cifras refieren no solamente al sector PYME, sino a la manufactura en general en los países listados. Sin embargo, datos presentados antes nos mostraron que la productividad (es decir el VAM por trabajador) es casi 4 veces mas baja en empresas más pequeñas, mientras los sueldos andan en un 51\% del promedio nacional. Eso significa que las pequeñas empresas tienen una posición aun más desfavorable frente a la competencia internacional.

Aparte de la productividad empresarial, el nivel de tecnología de un país también es un fuerte indicador de la competitividad internacional. No existen clasificaciones anuales sobre el nivel de tecnología por país, pero sí hay cifras de organizaciones multilaterales sobre este tema. Naciones Unidas, en 2001, clasificó a Nicaragua como "país marginalizado" en términos tecnológicos, la clasificación más baja que había.

La Organización de las Naciones Unidas del Desarrollo Industrial (ONUDI) publicó datos sobre competitividad en desempeño industrial en 1998, colocando a Nicaragua en el lugar 70 entre los 87 países, arriba de la mayoría de los países africanos, pero solamente superando a Paraguay en Latinoamérica. En 1985, la posición del país era 62. Confirmando esa mala clasificación del país en su capacidad de absorber y usar nueva tecnología está el "Networked Readiness Index" (Índice de Preparación de Redes) del Foro Económico Mundial, que mide el nivel en que un país está preparado y es capaz de utilizar los beneficios de las tecnologías de información y comunicación (ICT). En esta clasificación, Nicaragua se encuentra en posición 112, de un total de 115 países. Superando a Nicaragua están varios países africanos (Camerún, Malí, Kenya, Tanzania, Uganda), tal como países asiáticos más pobres como Bangladesh o Vietnam.

\section{Clima de negocios}

En los últimos años, organizaciones multilaterales como el Banco Mundial han prestado mucha atención al tema de 'clima de negocios', frecuentemente definido como el ámbito legal y jurídico en que empresas de un país tienen que operar. Elementos importantes del clima de negocios son: el peso administrativo de establecer un negocio, tiempo y costo para esforzar un contrato, facilidad de contratar y despedir trabajadores, presencia de corrupción etc. Por ende, este tema se trata más sobre el entorno del sector privado que sobre las actividades de las empresas mismas.

Gracias a la atención recibida, ahora existe una cantidad de información que permite comparar países y regiones en el mundo. La tabla de abajo presenta esta información, y prueba que el clima de negocios en Nicaragua es relativamente bueno. Es decir, la clasificación que tiene el país (59) en la lista es mejor que lo que se esperaría, basado en el PIB per cápita; Nicaragua se encuentra solamente dos posiciones bajo Panamá, y con mejor desempeño que países como Italia (70), México (73), El Salvador (76), Grecia (80), Costa Rica (89), China (91) y Brasil (117).

Una mirada a los indicadores detallados confirme esta idea. Nicaragua, en general, tiene relativamente bien organizados los procesos y el ámbito de sus negocios. La tabla muestra la comparación con México, El Salvador e Indonesia, con el fin de tratar de formar una idea 
del éxito del sector privado de aquellos países frente a Nicaragua.

Cuadro 3. Indicadores de Hacer Negocios en varios países y continentes, 2005.

\begin{tabular}{|c|c|c|c|c|c|c|c|}
\hline & Nicaragua & $\begin{array}{c}\text { EI } \\
\text { Salvador }\end{array}$ & México & Indonesia & $\begin{array}{l}\text { Latino- } \\
\text { américa }\end{array}$ & $\begin{array}{c}\text { Asía Oriente } \\
\text { \& Pacífico }\end{array}$ & OECD \\
\hline PIB por capita & 820 & 2,301 & 6,397 & 1,022 & & & \\
\hline Clasificación en facilidad de hacer negocios & 59 & 76 & 73 & 115 & & & \\
\hline \multicolumn{8}{|l|}{ Indicadores diversas } \\
\hline Iniciando un negocio (días) & 42 & 40 & 58 & 151 & 63 & 52.6 & 19.5 \\
\hline Dificultad de reclutar (index) & 11 & 44 & 33 & 61 & 42.5 & 26.5 & 30.1 \\
\hline Dificultad de despedir (index) & 50 & 20 & 60 & 70 & 31.9 & 23.0 & 27.4 \\
\hline Costos de despedir (semanas de sueldo) & 23.5 & 86 & 74.5 & 144.8 & 62.9 & 44.2 & 35.1 \\
\hline Protección del invertidor (index) & 5 & 4.7 & 3.7 & 5.3 & 4.5 & 5.3 & 5.9 \\
\hline Impuestos a pagar (\% utilidad bruto) & $54.3 \%$ & $32.2 \%$ & $31.3 \%$ & $38.8 \%$ & $52.8 \%$ & $31.2 \%$ & $45.4 \%$ \\
\hline Tiempo pagar impuestos (horas) & 240 & 224 & 536 & 560 & 529.3 & 249.9 & 197.2 \\
\hline Tiempo para exportar (días) & 38 & 43 & 18 & 25 & 30.3 & 25.8 & 12.6 \\
\hline Costos de esforzar contratos ( $\%$ deuda) & $16.3 \%$ & $12.5 \%$ & $20.0 \%$ & $126.5 \%$ & $23.3 \%$ & $61.7 \%$ & $10.6 \%$ \\
\hline Tiempo esforzar contratos (días) & 155 & 275 & 421 & 570 & 461.3 & 406.8 & 225.7 \\
\hline Tasa de recuperación (cerrando un negocio) & 33.9 & 30.5 & 64.1 & 13.1 & 28.2 & 24.0 & 73.8 \\
\hline
\end{tabular}

Fuente: Banco Mundial, Doing Business 2005

Aunque el ámbito legal de un país puede ser diseñado de forma adecuada, eso no siempre quiere decir que funcione en forma justa. El Banco Mundial deja la corrupción fuera de sus informes, y se enfoca en el diseño (más que en la ejecución) de la ley. Información estadística sobre corrupción en Nicaragua se puede encontrar en el índice global de la percepción de corrupción, mantenido por la organización Transparency International.

En su edición más reciente (2005), Nicaragua tuvo un grado de 2.6 (de 10), hallándose en posición 107 de un total de 159 países (tabla 8). El año anterior Nicaragua obtuvo el grado de 2.7, lo cual implica que entre 2004 y 2005 hubo cierto deterioro. Aunque sea marginal, eso es especialmente decepcionante, debido a la agenda de lucha contra la corrupción del presidente actual del país, Enrique Bolaños. Países que se destacan en sentido positivo aquí son Chile y El Salvador (menos corruptos, lo cual sugiere su nivel de PIB per cápita), mientras los países que se destacan en sentido negativo son Estados Unidos, Ecuador y Guatemala.

Si bien siempre existen aspectos que se pueden mejorar, la información de arriba nos llevaría a la conclusión de que la falta de productividad (y ergo competitividad) de las empresas nicaragüenses no está causada por un clima de negocios desfavorable. Sin embargo, la corrupción sí es un problema grave en Nicaragua; la igualdad entre las clasificaciones de corrupción y de competitividad de crecimiento (WEF) nos sugiere que la corrupción tiene un papel particularmente importante en definir la competitividad de un país y sus negocios. 
Cuadro 4. Percepción de corrupción, 2005.

\begin{tabular}{lcc} 
País & Posición & Grado (de 10) \\
\hline Icelandia & 1 & 9.7 \\
\hline Singapore & 5 & 9.4 \\
\hline Estados Unidos & 17 & 7.6 \\
\hline Chile & 21 & 7.3 \\
\hline Costa Rica & 51 & 4.2 \\
\hline El Salvador & 51 & 4.2 \\
\hline Mauritius & 51 & 4.2 \\
\hline Brasil & 62 & 3.7 \\
\hline México & 65 & 3.5 \\
\hline Panamá & 65 & 3.5 \\
\hline China & 78 & 3.2 \\
\hline India & 88 & 2.9 \\
\hline Honduras & 107 & 2.6 \\
\hline Nicaragua & 107 & 2.6 \\
\hline Zimbabwe & 107 & 2.6 \\
\hline Ecuador & 117 & 2.5 \\
\hline Guatemala & 117 & 2.5 \\
\hline Indonesia & 137 & 2.2 \\
\hline Bangladesh & 158 & 1.7 \\
\hline \hline
\end{tabular}

Fuente: Transparency International, 2005

\section{Competencia}

Otro tema importante en determinar el clima de inversiones de un país, y por ende su competitividad, es la competencia entre negocios locales. Más competencia significa más actividad empresarial, y más presión para mejorar productividad y calidad para los empresarios. Un sector privado dinámico generalmente está considerado como el motor más fuerte que puede empujar el desarrollo económico de un país.

Datos para medir la intensidad de la competencia nacional son difíciles a obtener. El Foro Económico Mundial, en la misma publicación que clasifica a los países en términos de su competitividad de crecimiento, usa un índice que mide la intensidad de competencia local, expresado en una cifra entre 1 y 7 . La tabla de abajo presenta estas cifras, y las compara con el PIB per cápita y el índice de competitividad de crecimiento (el GCI, Growth Competitiveness Index).

Los datos muestran que hay otra área en la cual Nicaragua tiene una posición muy desfavorable en el ámbito internacional. De todos los países mencionados, Nicaragua tiene el índice más bajo de intensidad de competencia (de hecho, el único país en el mundo con una cifra más baja es Angola, con un puntaje de 2.4). 
Cuadro 5. Intensidad de competencia local, $2004(1-7)$.

\begin{tabular}{|c|c|c|c|}
\hline & PBI p.c. & $\begin{array}{c}\text { WEF GCI } \\
2004\end{array}$ & $\begin{array}{l}\text { WEF index } \\
\text { comp. local } \\
2003 / 04\end{array}$ \\
\hline Estados Unidos & 37,425 & 2 & 5.9 \\
\hline Chile & 4,600 & 22 & 5.6 \\
\hline Jordan & 1,839 & 35 & 5.2 \\
\hline China* & 1,109 & 46 & 5.3 \\
\hline Mauritius* & 4,613 & 52 & 4.9 \\
\hline El Salvador & 2,249 & 53 & 5.0 \\
\hline México & 6,122 & 55 & 4.9 \\
\hline India & 555 & 55 & 5.6 \\
\hline Brazil & 2,788 & 57 & 5.2 \\
\hline Panamá & 4,124 & 58 & 4.5 \\
\hline Sri Lanka & 888 & 73 & 4.7 \\
\hline Indonesia & 958 & 74 & 4.0 \\
\hline Viet Nam & 476 & 77 & 4.9 \\
\hline Kenya & 439 & 78 & 5.2 \\
\hline Ecuador & 2,116 & 90 & 3.5 \\
\hline Honduras & 986 & 93 & 3.4 \\
\hline Nicaragua & 785 & 95 & 3.2 \\
\hline Zimbabwe & 388 & 99 & 3.6 \\
\hline Bangladesh & 419 & 102 & 4.8 \\
\hline Chad & 265 & 104 & 3.6 \\
\hline
\end{tabular}

Fuente: WEF (2005)

La baja intensidad de competencia en Nicaragua aporta a la explicación por su baja competitividad global. La tabla también demuestra la relación que existe entre el GCI y el índice de competencia local (entre más competencia local, más competitivo se vuelve un país). Esta observación lleva una conclusión fuerte para el gobierno de Nicaragua. Estimular la competencia en varios sectores del país debería ser una prioridad para su agenda política, con el fin de que se dinamice el sector privado, podría ser una estrategia efectiva para incrementar la competitividad del país, y, por ende, de sus empresas.

\section{CAFTA}

En el ámbito macroeconómico, el tratado de libre comercio entre los países centroamericanos y los Estados Unidos es un acontecimiento con consecuencias importantes para el futuro de los negocios nicaragüenses. El DR-CAFTA (Central American Free Trade Agreement) ha sido negociado con cinco países centroamericanos (El Salvador, Guatemala, Honduras, Nicaragua y Costa Rica) y la República Dominicana. Las negociaciones finalizaron oficialmente el 17 de diciembre de 2003, y el acuerdo fue ratificado por las asambleas de Estados Unidos y Nicaragua en el otoño de 2005.

El contenido del acuerdo pretende cambiar, en forma permanente, las reglas del comercio internacional entre los países en varios aspectos. Entre Nicaragua y EE.UU., el tratado primero eliminará los aranceles que todavía existen. Para los productos agrícolas (el área 
más sensible de las negociaciones) el plazo de reducción de los aranceles nicaragüenses será más largo (10-18 años), mientras que existen algunos productos (el azúcar, por ejemplo), cuya exportación quedará bajo un régimen de cuotas. El maíz blanco, producto crucial para la agricultura de subsistencia en Nicaragua, se queda fuera del tratado y mantiene sus aranceles. Vale notar que hasta ahora, un 90\% de las exportaciones desde Nicaragua ya están libres de aranceles bajo el Caribbean Basin Trade Protection Act (CBPTA).

Si bien las condiciones del tratado parecen balanceadas, es importante señalar que los productos agrícolas estadounidenses --que a través del tiempo verán importantes reducciones en sus aranceles en Nicaragua-- son fuertemente subsidiados por el gobierno de ese país. Por ende, se puede hablar de competencia injusta en el campo donde hay más que perder para las empresas nicaragüenses.

Nicaragua también liberará una variedad de productos industriales de EE.UU., actualmente con impuestos de 5 ó 10\% de aranceles, después la entrada en vigor del acuerdo. En su momento, Estados Unidos se ha comprometido a cancelar los impuestos aduaneros de $6 \%$ a las importaciones (el CBPTA ya había eliminado los aranceles).

Salvo aranceles, el DR-CAFTA también determina que los procedimientos aduaneros serán de acuerdo con las reglas de la Organización Mundial de Comercio (OMC), y la ejecución de ellos será transparente y eficiente. Además, Nicaragua ha comprometido la apertura de su sector de telecomunicaciones y finanzas para inversiones extranjeras. Por fin, la situación legal y la protección de inversiones extranjeras --anteriormente un reclamo frecuente de inversionistas internacionales-- serán mejoradas, y si se presentara el caso, un inversionista podría buscar mediación internacional si hubiera diferencias de interpretación. El acuerdo también asegura que un inversionista siempre recibirá un precio justo en caso de expropiación de bienes por el Estado.

\section{Situación actual}

Antes de evaluar el impacto del DR-CAFTA en las pequeñas empresas de Nicaragua, valdría la pena revisar la situación actual relacionada con la importación, exportación e inversiones extranjeras. En los capítulos anteriores ya vimos la composición del sector PYME de Nicaragua. En términos de productos, la exportación se trata predominantemente de carne bovina (17.6\% de exportaciones totales en 2002), café (11.8\%), camarones y langostas (5.6\%), oro (5.5\%), azúcar (4.8\%), maní (4.3\%), y frijol (3.0\%). La importación se concentró en petróleo y diesel (35.9\%), medicinas (9.3\%), vehículos (5.5\%), trigo (2.3\%) y concentrados para elaboración de aguas (2.1\%). En términos de su uso final, las importaciones fueron divididas entre bienes de consumo (32\% del valor CIF en 2002), productos petroleros (12\%), bienes intermedios (30\%) y bienes de capital (26\%).

Concentrándonos específicamente en el comercio con los Estados Unidos, se presentan las siguientes características: cifras de 2004 del Banco Central de Nicaragua, muestran que las exportaciones hacia Estados Unidos correspondían al 35\% de la exportación total (un monto de \$US263 millones). Los principales productos de exportación e importación se presentan abajo en dos diagramas. En la exportación se nota la importancia de los sectores pecuario 
(langostas, camarones y pescado), carne y café. En términos de manufacturera, sólo lozas sanitarias y cigarrillos se destacan como productos nicaragüenses en Estados Unidos.

La importación desde Estados Unidos (US\$492 millones o 22\% de la importación total), clasificada por uso final en vez de por producto, se caracteriza por una baja importación de petróleo (5\%) y un alto nivel de importación de los bienes de consumo no duraderos (34\%). Además, se nota que en bienes intermedios y de capital, el sector industria es mucho más importante (44\%) que el sector agropecuario (4\%). De hecho, dentro de las importaciones que no eran bienes de consumo sólo un 6.9\% fue dirigido al sector agropecuario, el resto se fue para transporte (y petróleo), industria y construcción.

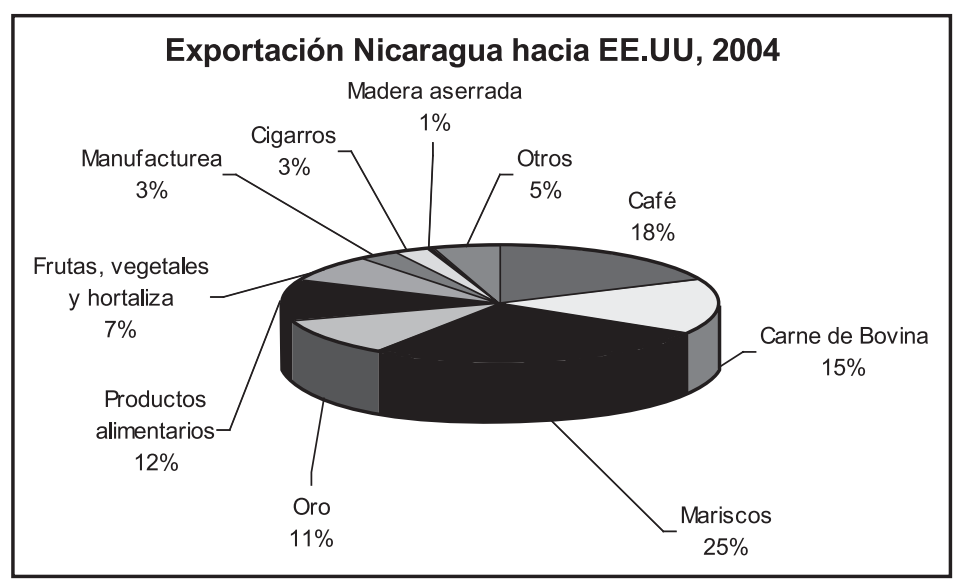

Ilustración 1. Distribución por rubro de exportación de Nicaragua hacia los Estados Unidos durante el 2004. Fuente: BCN, 2005 (sitio web)

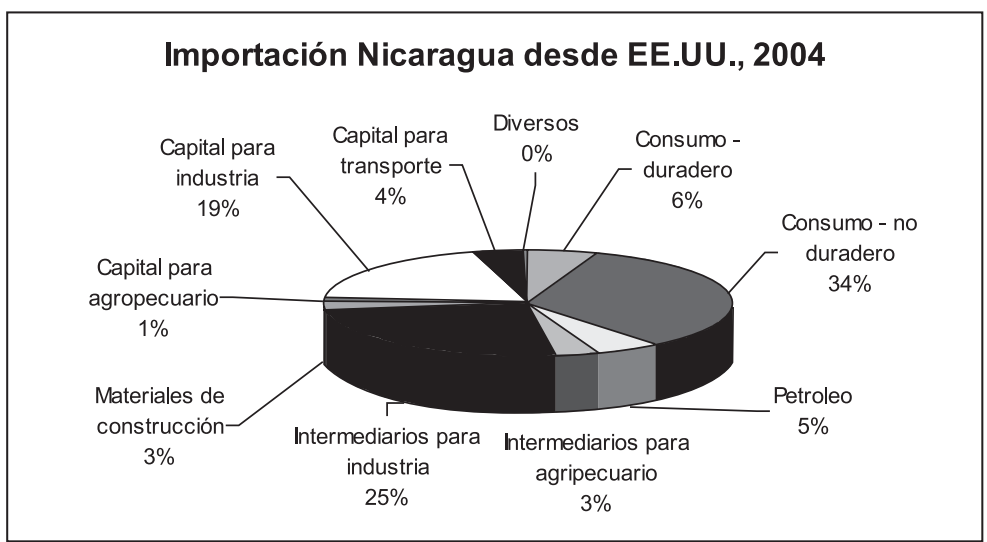

Ilustración 2. Distribución por rubro de importación desde los Estados Unidos hacia Nicaragua durante el 2004. Fuente: BCN, 2005 (sitio web) 


\section{Impacto para las PYMEs}

Datos del Banco Central de Nicaragua demuestran que las actividades de las pequeñas empresas en Nicaragua eran divido entre comercio (56\%), manufacturera (17\%), servicios sociales y comunitarios (11\%), hotelería (6\%), y otros (10\%). Esta división sugiere que la gran mayoría de las pequeñas empresas no serán afectadas por un auge de competencia por el CAFTA-DR, por el hecho que brindan servicios locales no-exportadores. Dado la importancia de bienes intermediarios en la importación, las pequeñas empresas pueden reducir sus costos y mejorar su competitividad con la reducción de aranceles en estos bienes. Además, existen oportunidades para crecer y vender más en el sector de comercio, y un potencial crecimiento general de la economía causará un incremento de la demanda general, incluso también para los productos y servicios de las pequeñas empresas. La competencia de los productos estadounidenses en el sector de la manufacturera será más fuerte, aunque la reducción de precio va por un máximo de $10 \%$.

Sin embargo, la información disponible se basa en el sector urbano, y no cuenta la gran cantidad de micro y pequeñas empresas agrícolas que existen. Allá, debido a la competencia "injusta" por los subsidios que agrega el gobierno estadounidense a sus productos, y a los actuales niveles de aranceles más altos, el peligro de un cambio y de un deterioro substancial para las pequeñas empresas es real. El plazo más largo de la reducción de aranceles, por lo tanto, es el único aspecto que puede proveer el tiempo necesario para que el sector se ajuste. El potencial de reducciones en los subsidios agrícolas de los países desarrollados, incluso los Estados Unidos, dentro de las negociaciones de la OMC y de la agenda de Doha, será tal vez la mejor oportunidad para balancear de nuevo la competitividad entre los sectores agrícolas de los dos países.

En términos de exportación, la cancelación de aranceles, en general, abre nuevas oportunidades para empresas interesadas en exportar. Aquí vale mencionar que las experiencias de otros países con el libre comercio, y más general con la globalización, también muestran que las empresas que ganan más son aquellas que cuentan con una mayor productividad y que poseen tecnologías más modernas. En breve, empresas que ya están más cerca al estilo y tipo de las de su mercado meta. Eso implicará que si ganarán empresas en Nicaragua, serán las empresas más avanzadas y productivas, por lo general, medianas o grandes. La tabla de abajo procura resumir los efectos para varios grupos económicos del país. La tabla no es amplia, y tampoco incluye una subdivisión por sector de las empresas. El impacto está basado en estimaciones del autor. 
Cuadro 7. Potencial impacto del CAFTA-DR en Nicaragua.

\begin{tabular}{|c|c|c|c|c|c|c|}
\hline $\begin{array}{l}\text { Efecto } \\
\text { CAFTA-DR }\end{array}$ & $\begin{array}{c}\text { Micro- } \\
\text { empresas }\end{array}$ & PyMEs & $\begin{array}{c}\text { Grandes } \\
\text { empresas }\end{array}$ & $\begin{array}{l}\text { Consumi- } \\
\text { dores Nic }\end{array}$ & $\begin{array}{c}\text { Empleo } \\
\text { Nic. }\end{array}$ & $\begin{array}{l}\text { Gobierno } \\
\text { Nic. }\end{array}$ \\
\hline $\begin{array}{l}\text { Reducción aranceles } \\
\text { agrícolas nicas }\end{array}$ & -- & -- & -- & + & -- & - \\
\hline $\begin{array}{l}\text { Reducción aranceles } \\
\text { industriales nicas }\end{array}$ & n.a. & $\begin{array}{l}\text { - } \boldsymbol{I +} \\
\text { (más comp., } \\
\text { (más ventas } \\
\text { comercio) }\end{array}$ & $\begin{array}{l}\text { - } \boldsymbol{I +} \\
\text { (más comp., } \\
\text { más ventas } \\
\text { comercio) }\end{array}$ & + & $-I+$ & - \\
\hline $\begin{array}{l}\text { Reducción aranceles } \\
\text { insumos nicas }\end{array}$ & + & + & + & + & + & - \\
\hline $\begin{array}{l}\text { Reducción costos y } \\
\text { tramites aduaneros } \\
\text { EE.UU. }\end{array}$ & n.a. & + & + & n.a. & + & n.a. \\
\hline $\begin{array}{l}\text { Apertura sector } \\
\text { telecomunicaciones nica }\end{array}$ & n.a. & $\begin{array}{c}\mathbf{+} \\
(\text { más } \\
\text { comercio })\end{array}$ & $\begin{array}{c}-\boldsymbol{I +} \\
\text { (comp., } \\
\text { comercio) }\end{array}$ & ++ & + & n.a. \\
\hline $\begin{array}{l}\text { Apertura sector finanzas } \\
\text { nica }\end{array}$ & n.a. & $\begin{array}{c}\mathbf{+} \\
\text { (oportun. } \\
\text { crédito y } \\
\text { exportación) }\end{array}$ & $\begin{array}{c}- \\
(\text { comp.) }\end{array}$ & ++ & + & $\begin{array}{c}\mathbf{+} \\
\text { (menos } \\
\text { riesgo } \\
\text { crises } \\
\text { financ.) }\end{array}$ \\
\hline $\begin{array}{l}\text { Reglas origen de } \\
\text { productos EE.UU. }\end{array}$ & n.a. & $\begin{array}{c}\mathbf{+} \\
\text { (oportunid. } \\
\text { exportación) }\end{array}$ & $\begin{array}{c}\mathbf{+} \\
\text { (oportunid. } \\
\text { exportación) }\end{array}$ & n.a. & + & n.a. \\
\hline $\begin{array}{l}\text { Mas inversión extranjera } \\
\text { (por fortalecimiento } \\
\text { posición legal) }\end{array}$ & $\begin{array}{c}- \\
(\mathrm{comp} .)\end{array}$ & (comp.) & $\begin{array}{c}- \\
(\text { comp. })\end{array}$ & ++ & $+I-$ & + \\
\hline $\begin{array}{l}\text { Más crecimiento } \\
\text { económico Nicaragua }\end{array}$ & $\begin{array}{c}\mathbf{+} \\
\text { (ventas) }\end{array}$ & $\begin{array}{c}\mathbf{+} \\
\text { (ventas) }\end{array}$ & $\begin{array}{c}\mathbf{+} \\
\text { (ventas) }\end{array}$ & n.a. & + & ++ \\
\hline
\end{tabular}

Fuente: estimaciones del autor

La tabla muestra que es difícil dar un juicio final sobre el impacto. En el sector de manufactura podrá haber cambios profundos causados por el aumento de competencia, mientras haya incrementos de actividad y empleo en el comercio por el mismo efecto del tratado. El grupo más afectado en sentido negativo parece ser el de las empresas agrícolas, mientras que los consumidores nicaragüenses ganan por todos los efectos del tratado.

Debido a que una gran cantidad de los microempresarios y empleados de las pequeñas y medianas empresas viven cerca del límite de la pobreza, éste también es un efecto positivo importante del acuerdo. Otro efecto amenazante puede ser la introducción de empresas extranjeras de comercio (los supermercados). En México se ha visto un cambio profundo en el porcentaje de ventas al público por pequeñas empresas hacia mayores ventas por grandes cadenas de 'outlets'. En Nicaragua, donde el índice de ventas por supermercado todavía es bajo, hay mucho potencial para cadenas de supermercados --sean nacionales o extranjeros--, de empezar a conquistar un porcentaje mayor de mercado, en ventas de productos de alimentación y otras necesidades primarias.

Los efectos más positivos del DR-CAFTA serían la reducción de aranceles de materias primas (insumos) y de los costos y trámites aduaneros. El crecimiento económico es un efecto secundario que no es garantizado, por ende no se puede considerar este efecto de la 
misma manera. De las 'nuevas' oportunidades de exportar, sólo se puede decir que éstas ya existen y han existido bajo el CBPTA, lo cual implica que un auge en la exportación no será un efecto muy probable de la vigencia del tratado.

El empleo nicaragüense parece ganar la gran mayoría de los efectos del acuerdo de libre comercio. Sin embargo, es importante notar que un solo gran efecto negativo (reducción del empleo agrícola) puede ser mucho más impactante que varios pequeños efectos positivos. El impacto de la facilitación de inversiones extranjeras depende de varios factores, y es casi imposible de prever. Algunos estudios pretenden comparar la situación centroamericana con la de México y el NAFTA, pero dadas las fuertes diferencias en términos de geografía, geopolítica, infraestructura y tamaño del mercado, esta comparación no parece muy prudente. De todas maneras, en México la mayoría de los análisis apunta al fuerte empuje de la inversión en nuevas fábricas y plantas cerca de la frontera, cosa que para las pequeñas empresas de Nicaragua no es muy relevante. Estudios sobre los "upward" y "downward linkages", refiriéndose a la manera en que nuevas grandes empresas proveen demanda para empresas locales más pequeñas, faltan o llegan a conclusiones ambiguas.

Resumiendo, el impacto del DR-CAFTA para el sector MIPYME en Nicaragua podrá existir, pero en este momento no hay muchas indicaciones que sugieran que el impacto será profundo en el corto y en el mediano plazos. Debido a la atención que han obtenido las negociaciones del DR-CAFTA en la prensa y en el país en general, tal vez se trata más que nada de un cambio de actitud, es decir, de un enfoque más fuerte en las posibilidades de exportación, que lleve la economía mundial a Nicaragua.

\section{El desafío de la globalización}

El comercio internacional ha pasado por un período de fuerte crecimiento sobre las últimas décadas. Este proceso de integración económica, y el proceso de desaparición de barreras para información, factores de producción y comercio entre naciones que lo acompañan, es conocido como globalización.

Desde las experiencias de una multitud de países en desarrollo, una de las principales conclusiones es que el proceso de globalización, ceteris paribus, no tiene un impacto positivo directo en el alivio de la pobreza. Hasta ahora, más bien, las diversas experiencias están sugiriendo que la globalización funciona como "desigualizador"; brinda nuevas oportunidades de crecimiento a empresas que saben manejar las demandas de los mercados internacionales, y al mismo tiempo pone presión competitiva adicional al sector de trabajo no calificado. Eso ha significado un crecimiento de las diferencias en pago entre trabajadores calificados y no calificados tanto a nivel nacional como internacional.

La solución para Nicaragua, particularmente para pequeñas empresas, se encontrará en insertarse en las cadenas de producción que se están generando por el proceso de globalización. Esta inserción no viene como 'maná del cielo', sino como algo que hay que trabajar. Elemento clave en el éxito de esta inserción es la competitividad de las empresas.

Nicaragua tiene una gran cantidad de problemas en este aspecto: el país es caro para producir 
(tiene niveles altos de sueldos en comparación con su PIB per cápita); la productividad laboral es bajísima (en el sector manufacturero por lo menos); la infraestructura no permite un transporte rápido y económico, agregando más costos a la producción; el sistema judicial sufre de corrupción; los niveles de educación son bajos; y no todos los empresarios están dispuestos a efectuar los cambios necesarios para responder de forma óptima a las demandas del mercado internacional.

No obstante todo esto, existen ejemplos de pequeñas empresas que sí han logrado encontrarse con mercados para sus productos, y oportunidades para vender y crecer. Existen varias oportunidades de integrar más de la cadena de producción agrícola en el propio país; hay muchas posibilidades de mejorar la calidad de la producción para que llegue a estándares internacionales, y varios sectores de servicios (por ejemplo turismo) tienen alto potencial de crecimiento. El cuadro siguiente presenta un excelente ejemplo de algunas PYMEs que lograron posicionarse bien frente las oportunidades de la globalización. 


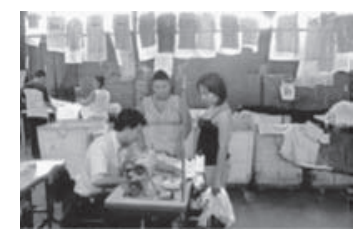

EFE / END. El esfuerzo de los pequeños textileros está dando frutos al ingresar a nichos de mercado en Estados Unidos y Puerto Rico.

\section{En preferencias arancelarias como las zonas francas Pequeños textileros entrando al mercado internacional}

\author{
* Beneficios fiscales sólo son para las empresas grandes, dice textilera \\ * Capacitación juega rol importante
}

De elaborar cuatro piezas al día con una vieja máquina de coser de pedal, Aida Patricia Mayorga, propietaria de Creaciones Oscarito's, es ahora una mediana empresaria de la rama textil vestuario, y a pesar de no gozar de los beneficios fiscales de las grandes empresas de zona franca, está exportando 20 mil prendas al mes a Estados Unidos y Puerto Rico.

Mayorga aseguró que su empresa, que ahora brinda empleo a 50 trabajadores, está cerca de entrar al régimen de zona franca, con lo que podrá gozar de los incentivos fiscales que hasta ahora sólo los han recibido los grandes empresarios.

Sin embargo, consideró que la mayoría de los pequeños y medianos negocios del sector, tienen que unir esfuerzos para tratar de lograr dichos incentivos. La empresaria aseguró que esperan beneficiarse de las preferencias arancelarias logradas en el Tratado de Libre Comercio entre República Dominicana, Centroamérica y Estados Unidos (DR-Cafta, por sus siglas en inglés) para el sector textil.

Dijo que ya cuentan con un representante en la Comisión Nacional de Textil Vestuario, para la repartición de dichas preferencias, conocidas como TPL, de manera que las pequeñas y medianas empresas del sector textil puedan aprovechar el Cafta. "Las zonas francas no pagan ningún impuesto, y nosotros debemos luchar para entrar a ese régimen y tener los mismos beneficios”, apuntó.

Mayorga dijo que el estar a punto de entrar al régimen de zona franca se debe a que ya cuenta con 50 empleados, lo que se cataloga como una mediana empresa, tiene edificio propio y está ubicado en una buena zona, en Carretera a Masaya. Dijo que el producto que exportan ha gustado mucho, y parte del logro se lo debe a la capacitación que ha recibido del Gobierno, lo que le ha permitido tecnificarse y producir con mejor calidad. La propietaria de Creaciones Oscarito's dijo que comenzó sin dinero, y por eso considera que la falta de recursos no es obstáculo para triunfar

\section{(Inpyme) minimiza incentivos de grandes}

Erwin Zelaya, Director General de Innovación y Mercadeo del Instituto de Apoyo a la Pequeña y Mediana Empresa (Inpyme), dijo que el reto que tiene esta institución es que los pequeños empresarios de la rama textil vestuario puedan aprovechar el Cafta.

Zelaya agregó que aunque las pequeñas empresas del sector textil vestuario no gocen de los incentivos del régimen de zonas francas, tienen la ventaja de poseer un nicho de mercado atractivo entre los consumidores nicas que viven en el extranjero.

"Siempre hay nichos de mercado. Tomemos en cuenta que el mercado americano es de consumo, lo que también da ventaja para los productos nostálgicos”, dijo.

Zelaya añadió que estas pequeñas empresas no tienen los beneficios de las zonas francas "porque no quieren". "Si se asocian, pueden optar a los beneficios de los grandes", expresó el funcionario. También indicó que de acuerdo con la capacidad de las pequeñas y medianas empresas, si logran colocar el producto en el mercado estadounidense, éste no tiene nada que ver con los que se elaboran en las zonas francas.

Zelaya reconoció que entre las pequeñas y medianas empresas del sector textil vestuario, ni siquiera se tiene mucha información, ya que no existe un censo sobre el sector. Dijo que muchas de estas Pymes están "invisibles", porque no tributan y no se tiene registros de sus operaciones. Consideró que se debe realzar la coordinación entre las instituciones públicas y las empresas privadas, para ayudar a mejorar la competitividad del sector. 


\section{Referencias bibliográficas}

-BANCO CENTRAL DE NICARAGUA (2006), Indicadores Económicos Mensuales [en línea], Managua, Nicaragua [consultado Feb-06], disponible en internet: http:/www.bcn.gob. ni/estadisticas/indicadores

-BANCO MUNDIAL (2002). Globalization, growth and poverty, Washington DC.

-BANCO MUNDIAL (2005). Doing Business, Washington DC.

-BANCO MUNDIAL (2005). Does Firm Size Matter?, Washington DC.

-BECK, T. and KUNT, A. (2003). SMEs, Growth and Poverty, Banco Mundial, Washington DC.

-BCN, Censo Industrial 2000 (sitio web)

-CEPAL (2001), Elementos de Competitividad Sistémica de las Pequeñas y Medianas

Empresas (PYME) del Istmo Centroamericano, LC/MEX/L.499, México.

-COMISIÓN SERVICIOS DE DESARROLLO EMPRESARIAL (MINISTERIO DE -FOMENTO, INDUSTRIA Y COMERCIO DEL GOBIERNO DE NICARAGUA)(2004). Matriz del sector Micro, pequeña y mediana empresa 2004-2008, Managua.

-FORO ECONÓMICO MUNDIAL (WEF, 2005). Global Competitiveness Report 2005-2006

[en linea], ed. Klaus Schwab, Davos, Switzerland, 28-Sep 2005 [consultado 13-Ene-06], Disponible en internet: http://www.weforum.org/gcr

-GTZ-INDE (2003). MIPYME en Nicaragua, Managua.

-INEC/MEDE/GTZ (1998). Análisis del censo económico urbano nacional, Managua.

-MAYORGA E. (2003). Lineamientos estratégicos del programa de fomento a la pequeña empresa, publicación centro MADE-UCA, Managua, Nicaragua

-NACIONES UNIDAS (2002). Human Development Report 2001, New York.

-ONUDI (2003). Industrial Development Report 2002/2003, Vienna.

-ONUDI (2003). Strategy document to enhance the contribution of an efficient and competitive SME sector to industrial and economic development in the Islamic republic of Iran, Teheran.

-ONUDI (2006). Selected Industrial Statistics [en linea], estadísticas en linea, ONUDI Vienna, Austria [consultado Feb-06], disponible in internet: http:/www.unido.org/data/ regions.cfm?area $=$ GLO

-ORGANIZACIÓN INTERNACIONAL DE TRABAJO (2006), LABORSTA Labour Statistics Database [en linea], ILO Turín, Italia [consultado Feb-06], disponible en internet: http:// www.ilo.org/public/english/bureau/stat/portal/online.htm

-PAIT, S. (2002). Estudio de Necesidades de Ampliación y mejoramiento de la oferta de servicios de desarrollo empresarial (SDE), y fortalecimiento institucional de los oferentes de SDE en Nicaragua, Managua.

-PORTER, M. (1990). Competitive Advantage of Nations, The Free Press, New York NY.

-SOLÓRZANO, J. (2000). La microempresa en Nicaragua en la década de los noventa, (publicación OIT), San José.

-SOLÓRZANO, J. (2003). Características y evolución reciente del mercado de trabajo en Nicaragua, organización Internacional de Trabajo (OIT), San José.

-TRANSPARENCY INTERNATIONAL (2006). Corruption Perceptions Index 2005 [en linea], TI Germany [consultado Feb-06], disponible en internet: http:/www.transparency.org/policy_ research/surveys_indices/cpi/2005

Feuismod oloborp erostio nsecte dipsum veliquisim vendions num in utat, vel euguerilitVullut 